\title{
MODELO EULERIANO SEMI-ANALÍTICO PARA A DISPERSÃO DE CONTAMINANTES NA CAMADA LIMITE PLANETÁRIA
}

Davidson M. Moreira, Angela B. D. Moura e Marco T. de Vilhena Universidade Federal do Rio grande do Sul Porto Alegre,RS - Brasil

Gervásio A. Degrazia

Departamento de Física - CCNE UFSM - Santa Maria, RS - Brasil

\section{RESUMO}

Um modelo de dispersão analítico baseado na discretização da Camada Limite Planetária em $N$ subcamadas é apresentado. Em cada subcamada a equação de difusão-adevcção é resolvida pelo emprego da transformada de Laplace, considerando um valor médio para o coeficiente de difusão e para a velocidade do vento. O presente modelo emprega perfis realísticos semi-empíricos para o coeficiente de difusão e velocidade do vento, de modo que a turbulência não homogênea pode ser utilizada. A performance do modelo é testada confrontando as simulações com os dados experimentais de Prairie Grass e Copenhagen. A aplicação de índices estatísticos (Hanna, 1989) mostra que o modelo analítico de dispersão proposto produz bons resultados. 


\section{ABSTRACT}

An analytical air quality dispersion model based on a discretization of the planetary boundary layer in $N$ sub-layers is presented. In each sub-layer the diffusion-advection equation is solved by the Laplace Transform techniques, considering an average value for the vertical exchange coefficient and the wind speed. As a consequence, the present approach allows to employ realistic semi-empirical profiles for the eddy diffusivity and wind speed, in such manner that the inhomogeneous turbulence can be handle. The model performance have been evaluated using the well-known Copenhagen and Prairie Grass datasets. Then, the application of the statistical evalution procedure (Hanna, 1989) over the out coming results has show that the proposed analytical dispersion model produces a good fitting of the observational data.

\section{INTRODUÇÃO}

A investigação da dispersão e do transporte de contaminantes na atmosfera é uma atividade fundamental na proteção da qualidade do ar. A partir da estimativa do campo de concentração de contaminantes próximo a fonte é possível avaliar o impacto ambiental causado e agir no sentido de solucionar o problema da forma mais conveniente.

A dificuldade em controlar as emissões de contaminantes liberados por diferentes fontes tem estimulado o desenvolvimento de modelos confiáveis que possam relacionar a taxa de emissão ao campo de concentração superficial. Com o objetivo de descrever adequadamente a relação entre as emissões e a qualidade do ar, um modelo deve levar em conta os parâmetros meteorológicos e os processos físicos que ocasionam a remoção de contaminantes. 
O estudo da dispersão de contaminantes na Camada Limite Convectiva (CLC) é especialmente importante no caso de emissões a partir de fontes elevadas, pois estas geralmente contribuem para a máxima concentração ao nível do solo durante a convecção. As altas concentrações ocorrem como resultado da forte mistura entre a superfície e o topo da CLC. Esta mistura, impulsionada pelo fluxo turbulento de calor positivo é responsável pelos perfis verticais homogêneos de concentração.

Neste trabalho a equação de difusão-advecção é resolvida analiticamente para a obtenção da concentração superficial de contaminantes na CLC. Motivado pela existência de dados observacionais, apresenta-se o estudo da dispersão bidimensional de contaminantes passivos abandonados por uma fonte linha contínua, onde a fonte é orientada perpendicularmente a direção do vento. No fechamento do fluxo turbulento de concentração vertical utiliza-se um coeficiente de difusão turbulenta, que emprega o modelo de difusão estatístico de Taylor e a teoria de similaridade convectiva.

\section{MODELO DE DISPERSÂO}

O estudo do transporte e dispersão de poluentes na atmosfera é muitas vezes descrito pela equação da difusão-advecção, que é obtida pela parametrização dos fluxos turbulentos na equação da continuidade pelo emprego do modelo de transporte por gradiente ou teoria $K$. Para um sistema de coordenadas cartesiano em que a direção $x$ coincide com a do vento médio, a equação da difusão-advecção no estado estacionário é escrita como (Arya, 1994): 


$$
U \frac{\partial \bar{c}}{\partial x}=\frac{\partial}{\partial x}\left(K_{x} \frac{\partial \bar{c}}{\partial x}\right)+\frac{\partial}{\partial y}\left(K_{y} \frac{\partial \bar{c}}{\partial y}\right)+\frac{\partial}{\partial z}\left(K_{z} \frac{\partial \bar{c}}{\partial z}\right)
$$

onde $\bar{c}$ representa a concentração média, $U$ o vento médio na direção $x$ e $K_{x}$ , $K_{y}$ e $K_{z}$ são os coeficientes de difusão turbulenta nas direções $x, y$ e $z$, respectivamente. Integrando-se lateralmente a equação (1) e desprezandose a difusão longitudinal resulta:

$$
U \frac{\partial \overline{c^{y}}}{\partial x}=\frac{\partial}{\partial z}\left(K_{z} \frac{\partial \overline{c^{y}}}{\partial z}\right)
$$

sujeita as seguintes condições de contorno:

$$
\begin{gathered}
U_{z} \overline{c^{y}}(0, z)=Q \delta\left(z-H_{s}\right) \\
K_{z} \frac{\partial \overline{c^{y}}}{\partial z}=0 \quad \text { em } \quad z=0, z_{i}
\end{gathered}
$$

onde $\overline{c^{y}}$ representa a concentração média integrada lateralmente, $H_{s}$ a altura da emissão e $Q$ a intensidade da fonte.

Levando-se em consideração a dependência do coeficiente $K_{z}$ e o perfil do vento $U$ sobre a variável $z$, a altura $z_{i}$ da CLC é discretizada em $N$ subintervalos. Cada intervalo de $K_{z}$ e de $U$ assume o valor médio:

$$
K_{n}=\frac{1}{z_{n}-z_{n-1}} \int_{z_{n-1}}^{z_{n}} K_{z}(z) d z \quad ; \quad U_{n}=\frac{1}{z_{n}-z_{n-1}} \int_{z_{n-1}}^{z_{n}} U(z) d z
$$

Portanto, a solução da equação (2) é reduzida a solução de $N$ problemas do tipo:

$$
U_{n} \frac{\partial}{\partial x} \overline{c_{n}^{y}}=K_{n} \frac{\partial^{2}}{\partial z^{2}} \overline{c_{n}^{y}} \quad \text { com } \quad \mathrm{zn}-1 \leq \mathrm{z} \leq \mathrm{z}_{\mathrm{n}}
$$


para $n=1$ : $N$, onde $\overline{c_{n}^{y}}$ representa a concentração no $n^{\text {th }}$ subintervalo. Para determinar as $2 N$ constantes de integração, a condição adicional (2N-2), chamada de continuidade da concentração e fluxo na interface são consideradas:

$$
\begin{gathered}
\overline{c_{n}^{y}}=\overline{c_{n+1}^{y}} \quad z=z_{n} \quad n=1,2, \ldots(\mathrm{N}-1) \\
K_{n} \frac{\partial \overline{c_{n}^{y}}}{\partial z}=K_{n+1} \frac{\partial \overline{c_{n+1}^{y}}}{\partial z} \quad z=z_{n} \quad n=1,2, \ldots(\mathrm{N}-1)
\end{gathered}
$$

Aplicando-se a transformada de Laplace na equação (5) resulta:

$$
\frac{\partial^{2}}{\partial z^{2}} \overline{c_{n}^{y}}(s, z)-\frac{U_{n} s}{K_{n}} \overline{c_{n}^{y}}(s, z)=-\frac{U_{n}}{K_{n}} \overline{c_{n}^{y}}(0, z)
$$

onde $\overline{c_{n}^{y}}(s, z)=L\left\{\overline{c_{n}^{y}}(x, z) ; x \rightarrow s\right\}$, que tem a conhecida solução:

$$
\overline{c_{n}^{y}}(s, z)=A_{n} e^{-R_{n} z}+B_{n} e^{R_{n} z}+\frac{Q}{2 R_{a}}\left(e^{-R_{n}\left(z-H_{s}\right)}-e^{R_{n}\left(z-H_{s}\right)}\right)
$$

onde,

$$
R_{n}= \pm \sqrt{\frac{U_{n} s}{K_{n}}} \quad \text { e } \quad R_{a}= \pm \sqrt{U_{n} s K_{n}}
$$

Finalmente, aplicando-se as condições de contorno e interface é obtido um sistema linear para as constantes de integração. A concentração é obtida invertendo-se numericamente a concentração transformada $\bar{c}$ por quadratura Gaussiana (Heydarian and Mullineaux, 1989):

$$
\overline{c_{n}^{y}}(x, z)=\sum_{j=1}^{N i} A_{j} \frac{P_{j}}{x}\left(A_{n} e^{-\sqrt{\frac{P_{j} U_{n}}{x K_{n}}} z}+B_{n} e^{\sqrt{\frac{P_{j} U_{n}}{x K_{n}}} z}\right)
$$




$$
\begin{gathered}
\overline{c_{n}^{y}}\left(x, z^{\prime}\right)=\sum_{j=1}^{N i} A_{j} \frac{P_{j}}{x}\left[A_{n} e^{-\sqrt{\frac{P_{j} U_{n}}{x K_{n}} z}}+B_{n} e^{\sqrt{\frac{P_{j} U_{n}}{x K_{n}}} z}+\right. \\
\left.+\frac{1}{2} \frac{Q}{\sqrt{\frac{P_{j} K_{n} U_{n}}{x}}}\left(e^{-\left(z^{\prime}-H_{s}\right) \sqrt{\frac{P_{j} U_{n}}{x K_{n}}}}-e^{\left(z^{\prime}-H_{s}\right) \sqrt{\frac{P_{j} U_{n}}{x K_{n}}}}\right)\right]
\end{gathered}
$$

onde $z^{\prime}$ é a região da altura da fonte. A solução nesta região é a equação (10b). Fora desta região a solução é a equação (10a). $A_{n}$ e $B_{n}$ são as constante de integração que são determinadas aplicando-se as condições de contorno e interface; $A_{j}$ e $P_{j}$ são, respectivamente, os pesos e pontos da quadratura Gaussiana. Neste estudo, foi considerado $N i=8$, sendo que este valor produz resultados mais precisos.

\section{DADOS EXPERIMENTAIS E PARAMETRIZAÇÃO METEOROLÓGICA}

A performance do modelo foi comparada com dados experimentais de Prairie Grass (Barad, 1958) e Copenhagem (Gryning, 1981). Para o experimento de Prairie Grass temos uma fonte pontual na altura de $\approx 0.5 \mathrm{~m}$. As concentrações médias foram coletadas a uma altura de $1.5 \mathrm{~m}$ em cinco distâncias da fonte (50, 100, 200, 400 e 800 m). Apresentase aqui o resultado para 20 experimentos convectivos, onde a condição $Z_{i} / L>10$ é satisfeita. No experimento de Copenhagen o traçador $\mathrm{SF}_{6}$ foi liberado de uma altura de $115 \mathrm{~m}$, e coletado no solo. As condições meteorológicas durante o experimento de Copenhagen estão na tabela 1 , enquanto que para o experimento de Prairie Grass na tabela 3.

A parametrização no modelo das eqs. (10a) e (10b) necessita de perfis verticais para difusividade turbulenta e vento médio. $O$ coeficiente 
de difusão $K_{z}$ é apresentado na forma adimensional como um perfil de similaridade, usando a escala de velocidade convectiva $\mathcal{W}_{*}$ e a altura da camada limite $z_{i}$. A expressão para $K_{z}$ emprega uma relação empírica entre a estabilidade e o maior comprimento de onda para o espectro da energia cinética turbulenta obtido de dados experimentais (Caughey and Palmer, 1979) e assume a simples forma derivada por (Degrazia et al., 1995):

$$
\frac{K_{z z}}{w_{*} z_{i}}=0,22\left(\frac{z}{z_{i}}\right)^{1 / 3}\left(1-\frac{z}{z_{i}}\right)^{1 / 3}\left[1-\exp \left(-\frac{4 z}{z_{i}}\right)-0,0003 \exp \left(\frac{8 z}{z_{i}}\right)\right]
$$

O perfil do vento médio tem sido parametrizado seguindo a Teoria de Similaridade de Monin-Obukhov e o modelo OML (Berkowicz et al., 1986):

$$
\begin{gathered}
U=\frac{u_{*}}{k}\left[\ln \left(z / z_{0}-\Psi_{m}(z / L)+\Psi_{m}\left(z_{0} / L\right)\right] \quad \text { se } z \leq z_{b}\right. \\
U=U\left(z_{b}\right) \quad \text { se } z>z_{b}
\end{gathered}
$$

onde $z_{b}=\min \left[|L|, 0,1 z_{i}\right]$, e $\Psi_{m}$ é a função estabilidade dada por (Paulsen, 1975):

$$
\begin{gathered}
\Psi_{m}=2 \ln \left[\frac{1+A}{2}\right]+\ln \left[\frac{1+A^{2}}{2}\right]-2 \tan ^{-1} A+\frac{\pi}{2} \\
A=(1-16 z / L)^{1 / 4}
\end{gathered}
$$

$k=0,4$ é a constante de Von Karman, $u_{*}$ é a velocidade de fricção e $z_{0}$ o comprimento de rugosidade aerodinâmico.

Nas tabelas 2 e 3 estão os resultados comparativos dos dados observacionais e do modelo para os experimentos de Copenhagen e Prairie Grass, respectivamente. A tabela 4 apresenta os índices estatísticos da performance do modelo. 
Tabela 1: Condições meteorológicas durante o experimento de Copenhagen $\left(\mathrm{H}_{\mathrm{s}}=115 \mathrm{~m}\right)$.

\begin{tabular}{cccccc}
\hline Exp. & $\begin{array}{c}U \\
\left(\mathrm{~ms}^{-1}\right)\end{array}$ & $\begin{array}{c}u^{*} \\
\left(\mathrm{~ms}^{-1}\right)\end{array}$ & $\begin{array}{c}L \\
(\mathrm{~m})\end{array}$ & $\begin{array}{c}W^{*} \\
\left(\mathrm{~ms}^{-1}\right)\end{array}$ & $\begin{array}{c}\mathrm{Z}_{i} \\
(\mathrm{~m})\end{array}$ \\
\hline 1 & 3,4 & 0,36 & -37 & 1,8 & 1980 \\
2 & 10,6 & 0,73 & -292 & 1,8 & 1920 \\
3 & 5,0 & 0,38 & -71 & 1,3 & 1120 \\
4 & 4,6 & 0,38 & -133 & 0,7 & 390 \\
5 & 6,7 & 0,45 & -444 & 0,7 & 820 \\
6 & 13,2 & 1,05 & -432 & 2,0 & 1300 \\
7 & 7,6 & 0,64 & -104 & 2,2 & 1850 \\
8 & 9,4 & 0,69 & -56 & 2,2 & 810 \\
9 & 10,5 & 0,75 & -289 & 1,9 & 2090 \\
\hline
\end{tabular}

Tabela 2: Concentração $\overline{c^{y}}(x, 0) / Q$ observada e simulada pelo modelo usando os dados do experimento de Copenhagen.

120 Rev. Ciência e Natura, Dispersion Process: 113 - 126 , 2000. 


\begin{tabular}{|c|c|c|c|}
\hline Exp. & $\begin{array}{l}\text { Distância } \\
\text { (m) }\end{array}$ & $\begin{array}{l}\text { Observada } \\
\left(10^{-4} \mathrm{~s} / \mathrm{m}^{2}\right)\end{array}$ & $\begin{array}{c}\text { modelo } \\
\left(10^{-4} \mathrm{~s} / \mathrm{m}^{2}\right)\end{array}$ \\
\hline \multirow[t]{2}{*}{1} & 1900 & 6,48 & 7,15 \\
\hline & 3700 & 2,31 & 4,12 \\
\hline \multirow[t]{2}{*}{2} & 2100 & 5,38 & 3,95 \\
\hline & 4200 & 2,95 & 2,82 \\
\hline \multirow[t]{3}{*}{3} & 1900 & 8,20 & 8,25 \\
\hline & 3700 & 6,22 & 5,62 \\
\hline & 5400 & 4,30 & 4,25 \\
\hline 4 & 4000 & 11,66 & 8,02 \\
\hline \multirow[t]{3}{*}{5} & 2100 & 6,72 & 7,05 \\
\hline & 4200 & 5,84 & 5,77 \\
\hline & 6100 & 4,97 & 4,75 \\
\hline \multirow[t]{3}{*}{6} & 2000 & 3,96 & 2,75 \\
\hline & 4200 & 2,22 & 2,22 \\
\hline & 5900 & 1,83 & 1,82 \\
\hline \multirow[t]{3}{*}{7} & 2000 & 6,70 & 4,75 \\
\hline & 4100 & 3,25 & 3,17 \\
\hline & 5300 & 2,23 & 2,60 \\
\hline \multirow[t]{3}{*}{8} & 1900 & 4,16 & 4,57 \\
\hline & 3600 & 2,02 & 3,20 \\
\hline & 5300 & 1,52 & 2,45 \\
\hline \multirow[t]{3}{*}{9} & 2100 & 4,58 & 3,65 \\
\hline & 4200 & 3,11 & 2,50 \\
\hline & 6000 & 2,59 & 1,90 \\
\hline
\end{tabular}


Tabela 3: Parâmetros de Praire Grass. $\overline{c^{y}}(x, 1.5)\left(\mathrm{g} \cdot \mathrm{m}^{-2}\right)$ na primeira linha para Praire Grass, na segunda linha, o presente modelo $\left(H_{s}=\right.$ $0,5 \mathrm{~m})$.

\begin{tabular}{|c|c|c|c|c|c|c|c|c|c|c|}
\hline Exp. & $\begin{array}{l}-L \\
(\mathrm{~m})\end{array}$ & $\begin{array}{c}z_{i} \\
(\mathrm{~m})\end{array}$ & $\begin{array}{c}W^{*} \\
(\mathrm{~m} / \mathrm{s})\end{array}$ & $\begin{array}{c}U \\
(\mathrm{~m} / \mathrm{s})\end{array}$ & $\begin{array}{c}Q \\
(\mathrm{~g} / \mathrm{s})\end{array}$ & $\begin{array}{l}50 \\
(\mathrm{~m})\end{array}$ & $\begin{array}{l}100 \\
(\mathrm{~m})\end{array}$ & $\begin{array}{l}200 \\
(\mathrm{~m})\end{array}$ & $\begin{array}{l}400 \\
(\mathrm{~m})\end{array}$ & $\begin{array}{l}800 \\
(\mathrm{~m})\end{array}$ \\
\hline \multirow[t]{2}{*}{1} & 9 & 260 & 0,84 & 3,2 & 82 & 7,0 & 2,3 & 0,51 & 0,16 & 0,062 \\
\hline & & & & & & 6,8 & 4,3 & 2,51 & 0,94 & 0,400 \\
\hline \multirow[t]{2}{*}{5} & 28 & 780 & 1,64 & 7,0 & 78 & 3,3 & 1,8 & 0,81 & 0,29 & 0,092 \\
\hline & & & & & & 4,0 & 2,5 & 1,53 & 0,77 & 0,170 \\
\hline \multirow[t]{2}{*}{7} & 10 & 1340 & 2,27 & 5,1 & 90 & 4,0 & 2,2 & 1,0 & 0,40 & 0,18 \\
\hline & & & & & & 4,6 & 2,8 & 1,6 & 0,62 & 0,22 \\
\hline \multirow[t]{2}{*}{8} & 18 & 1380 & 1,87 & 5,4 & 91 & 5,1 & 2,6 & 1,1 & 0,39 & 0,14 \\
\hline & & & & & & 5,3 & 3,2 & 1,9 & 0,92 & 0,10 \\
\hline \multirow[t]{2}{*}{9} & 31 & 550 & 1,70 & 8,4 & 92 & 3,7 & 2,2 & 1,0 & 0,41 & 0,13 \\
\hline & & & & & & 4,0 & 2,5 & 1,5 & 0,79 & 0,20 \\
\hline \multirow[t]{2}{*}{10} & 11 & 950 & 2,01 & 5,4 & 92 & 4,5 & 1,8 & 0,71 & 0,20 & 0,032 \\
\hline & & & & & & 4,7 & 2,9 & 1,75 & 0,69 & 0,240 \\
\hline \multirow[t]{2}{*}{15} & 8 & 80 & 0,70 & 3,8 & 96 & 7,1 & 3,4 & 1,35 & 0,37 & 0,11 \\
\hline & & & & & & 7,3 & 4,8 & 2,84 & 1,29 & 0,48 \\
\hline \multirow[t]{2}{*}{16} & 5 & 1060 & 2,03 & 3,6 & 93 & 5,0 & 1,8 & 0,48 & 0,10 & 0,017 \\
\hline & & & & & & 5,6 & 3,5 & 1,74 & 0,36 & 0,027 \\
\hline \multirow[t]{2}{*}{19} & 28 & 650 & 1,58 & 7,2 & 102 & 4,5 & 2,2 & 0,86 & 0,27 & 0,058 \\
\hline & & & & & & 5,2 & 3,2 & 1,96 & 0,98 & 0,230 \\
\hline \multirow[t]{2}{*}{20} & 62 & 710 & 1,92 & 11,3 & 102 & 3,4 & 1,8 & 0,85 & 0,34 & 0,13 \\
\hline & & & & & & 4,1 & 2,4 & 1,56 & 0,92 & 0,33 \\
\hline \multirow[t]{2}{*}{25} & 6 & 650 & 1,35 & 3,2 & 104 & 7,9 & 2,7 & 0,75 & 0,30 & 0,063 \\
\hline & & & & & & 7,8 & 4,8 & 2,48 & 0,64 & 0,140 \\
\hline
\end{tabular}


Tabela 3: continuação...

\begin{tabular}{|c|c|c|c|c|c|c|c|c|c|c|}
\hline \multirow[t]{2}{*}{26} & 32 & 900 & 1,86 & 7,8 & 98 & 3,9 & 2,2 & 1,04 & 0,39 & 0,127 \\
\hline & & & & & & 4,2 & 2,8 & 1,80 & 0,96 & 0,260 \\
\hline \multirow[t]{2}{*}{27} & 30 & 1280 & 2,08 & 7,6 & 99 & 4,3 & 2,3 & 1,16 & 0,46 & 0,176 \\
\hline & & & & & & 4,7 & 2,9 & 1,79 & 1,02 & 0,240 \\
\hline \multirow[t]{2}{*}{30} & 39 & 1560 & 2,23 & 8,5 & 98 & 4,2 & 2,3 & 1,11 & 0,40 & 0,10 \\
\hline & & & & & & 4,6 & 2,7 & 1,77 & 0,99 & 0,32 \\
\hline \multirow[t]{2}{*}{43} & 16 & 600 & 1,66 & 6,1 & 99 & 5,0 & 2,4 & 1,09 & 0,37 & 0,12 \\
\hline & & & & & & 5,0 & 3,1 & 1,85 & 0,81 & 0,07 \\
\hline \multirow[t]{2}{*}{44} & 25 & 1450 & 2,20 & 7,2 & 101 & 4,5 & 2,3 & 1,09 & 0,43 & 0,14 \\
\hline & & & & & & 4,9 & 2,9 & 1,83 & 0,92 & 0,20 \\
\hline \multirow[t]{2}{*}{49} & 28 & 550 & 1,73 & 8,0 & 102 & 4,3 & 2,4 & 1,16 & 0,45 & 0,15 \\
\hline & & & & & & 4,5 & 2,7 & 1,70 & 0,85 & 0,20 \\
\hline \multirow[t]{2}{*}{50} & 26 & 750 & 1,91 & 8,0 & 103 & 4,2 & 2,3 & 0,91 & 0,39 & 0,11 \\
\hline & & & & & & 4,6 & 2,8 & 1,82 & 0,94 & 0,22 \\
\hline \multirow[t]{2}{*}{51} & 40 & 1880 & 2,30 & 8,0 & 102 & 4,7 & 2,4 & 1,00 & 0,38 & 0,084 \\
\hline & & & & & & 4,9 & 2,9 & 1,86 & 1,05 & 0,340 \\
\hline \multirow[t]{2}{*}{61} & 38 & 450 & 1,65 & 9,3 & 102 & 3,5 & 2,1 & 1,14 & 0,53 & 0,20 \\
\hline & & & & & & 4,2 & 2,6 & 1,62 & 0,86 & 0,26 \\
\hline
\end{tabular}

Tabela 4: Índices estatísticos da performance do modelo.

\begin{tabular}{ccccc}
\hline Experimento & Nmse & Fa2 & Cor & Fb \\
\hline Copenhagen & 0,07 & 1,00 & 0,90 & 0,058 \\
Prairie Grass & 0,09 & 0,98 & 0,61 & $-0,205$ \\
\hline
\end{tabular}


Uma análise dos resultados mostra uma boa concordância entre as concentrações observadas e as simuladas pelo modelo. Para uma análise mais precisa foram aplicados os seguintes índices estatísticos (Hanna, S.R., 1989):

Nmse (normalized mean square error) $=\overline{\left(C_{o}-C_{p}\right)^{2}} / \overline{C_{o} C_{p}}$, informa sobre todos os desvios entre as concentrações simuladas pelo modelo e as observadas. É uma estatística adimensional e seu valor deve ser o menor possível para um bom modelo.

$\mathrm{Fa} 2=$ fraction of data $(\%)$ for $0.5 \leq\left(C_{p} / C_{o}\right) \leq 2$

Cor (correlation coefficient) $=\overline{\left(C_{o}-\overline{C_{o}}\right)\left(C_{p}-\overline{C_{p}}\right.} / \sigma_{o} \sigma_{p}$, descreve o grau de associação ou concordância entre as variáveis. Para um boa performance o seu valor deve ser 1 .

$\mathrm{Fb}$ (fractional bias) $=\overline{C_{o}}-\overline{C_{p}} / 0.5\left(\overline{C_{o}}+\overline{C_{p}}\right)$, informa a tendência do modelo de sobrestimar ou subestimar as concentrações observadas. $O$ valor ótimo é zero.

Em todas as expressões $C_{o}$ e $C_{m}$ são as concentrações observadas e as simuladas pelo modelo, respectivamente, e $\sigma$ é o desvio padrão.

\section{CONCLUSÕES}

Este trabalho descreve o desenvolvimento e o teste de um modelo analítico que estima a dispersão de contaminantes em uma CLP. O modelo é baseado na solução da equação de difusão-advecção pelo emprego da transformada de Laplace. A CLP é discretizada em $N$ subintervalos de modo que em cada subintervalo o coeficiente de difusão e o vento assumem um valor médio constante.

124 Rev. Ciência e Natura, Dispersion Process: 113 - 126 , 2000. 
O modelo utiliza perfis semi-empíricos para o coeficiente de difusão e velocidade do vento, de modo que a turbulência não homogênea pode ser descrita. Uma análise dos resultados e a aplicação dos índices estatísticos mostram que o modelo de dispersão turbulenta, com a parametrização utilizada, produzem bons resultados. Portanto, este confronto preliminar sugere que o modelo de dispersão turbulenta dado pelas eqs. (10a) e (10b) pode ser aplicado em um modelo de dispersão de contaminantes na atmosfera.

O presente trabalho foi realizado com o apoio do CNPq e CAPES, entidades do Governo Brasileiro voltadas ao desenvolvimento científico e tecnológico.

\section{REFERÊNCIAS}

Arya, P.S. (1994). Modelling and parameterization of near-source diffusion in weak winds. J. Appl. Met., 34, 1112-1122.

Barad, M.L. Project Prairie Grass, Geophys. Res. Pap. No 59, 1 and 2 (1958). Berkowicz, R.R., Olesen, H.R. and Torp U. (1986). The Danish Gaussian air pollution model (OML): Description, test and sensitivity analysis in view of regulatory applications. Air Pollution modeling and its application. pp. 453480. Edited by C. De Wispeleare, F.A. Schiermeirier and N.V. Gillani. Plenum Publishing Corporation.

Caughey, S. J. and Palmer, S.G. (1979). Some aspects of turbulence structure through of depth of the convective boundary layer. Quart. J. R. Met. Soc. 105, 811-827. 
Degrazia, G.A., Moraes, O.L.L. and Oliveira, A.P. (1995). An analytical formulation for mixing length scales in a convective PBL. Procedings of $21^{\text {th }}$ Int. Technical Meeting on Air Pollution and its Application, 6-10 Nov. 1995, Baltimore (US).

Gryning, S.E. (1981). Elevated source SF6 - tracer dispersion experiments in the Copenhagen area. Report RISOE-R-446, Risoc National Laboratory, Roskilde, Denmark.

Hanna, S.R. (1989). Confidence limit for air quality models as estimated by bootstrap and jacknife resampling methods. Atmospheric Environment, 23, 1385-1395.

Heydarian, M. and Mullineaux, N. (1989). Solution of parabolic partial differential equations. Appl. Math. Modelling, 5, 448-449.

Paulsen, C.A. (1975). The mathematical representation of wind and temperature profiles in a unstable atmospheric surface layer. J. Appl. Met., 9, 857-861. 